\title{
STIL: a multi-function protein required for dopaminergic neural proliferation, protection, and regeneration
}

\author{
Lei Li ${ }^{1,2}$, Congcong Liu ${ }^{2}$ and Aprell L. Carr ${ }^{1}$
}

\begin{abstract}
Degeneration of dopaminergic (DA) neurons in the brain is the major cause for Parkinson's disease (PD). While genetic loci and cellular pathways involved in DA neuron proliferation have been well documented, the genetic and molecular and cellular basis of DA cell survival remains to be elucidated. Recently, studies aimed to uncover the mechanisms of DA neural protection and regeneration have been reported. One of the most recent discoveries, i.e., multi-function of human oncogene SCL/TAL interrupting locus (Sti) in DA cell proliferation, neural protection, and regeneration, created a new field for studying DA cells and possible treatment of PD. In DA neurons, Stil functions through the Sonic hedgehog (Shh) pathway by releasing the inhibition of SUFU to GLII, and thereby enhances Shh-target gene transcription required for neural proliferation, protection, and regeneration. In this review article, we will highlight some of the new findings from researches relate to Stil in DA cells using zebrafish models and cultured mammalian PC12 cells. The findings may provide the proof-of-concept for the development of Stil as a tool for diagnosis and/or treatment of human diseases, particularly those caused by DA neural degeneration.
\end{abstract}

\section{FACTS}

- Stil is a human oncogene which may also function as $\mathrm{ON}-\mathrm{OFF}$ switches that regulate the death and survival of neurons

- Stil is required for neural proliferation and regeneration

- Stil-mediated Shh signaling transduction protects dopaminergic neurons in response to toxic drug treatment

\section{OPEN QUESTIONS}

- How to control the expression of Stil mRNA in neurons?

\footnotetext{
Correspondence: Lei Li (Li.78@nd.edu)

'Department of Biological Sciences, University of Notre Dame, Notre Dame, IN 46556, USA

${ }^{2}$ Center for Reproductive Medicine, Shandong University, Jinan 250001, China Edited by R. Killick
}

- Does Stil play a role in regulation of neural function?

- Can Stil be developed as a molecular marker for diagnosis of neural degeneration?

\section{Introduction}

The SCL/TAL interrupting locus (Stil) is a human oncogene that was originally identified from human $\mathrm{T}$-cell lymphoblastic leukemia, where it functions as a hematopoietic transcription factor ${ }^{1}$. The sequence of Stil gene is highly conserved in vertebrate species ${ }^{2}$. In humans, the Stil transcript is comprised of 18 exons which yield a peptide of 1287 amino acids in $143-\mathrm{KD}^{2,3}$. While STIL does not share any known structural motifs with other proteins, analyses of the human STIL sequence revealed some similarities between STIL and the C-terminus of cytokine TGF- $\beta^{4}$ and the C-terminus of centriole duplication factor Ana2 $2^{5}$. STIL is expressed in all cell types examined so far ${ }^{6-10}$. In prometaphase-synchronized cells and mouse tumor cell lines, STIL functions as a cell cycle checkpoint protein that regulates the transition of mitotic 
entry during cell proliferation ${ }^{11}$. In developing human cancer cells, STIL is localized to the pericentriolar region of the centrosomes, where it regulates in spindle pole positioning as well as centriole formation and duplication $^{12-14}$.

While ample proof of STIL functions in cancer can be found in the literature, evidence also exists that STIL is involved in the development and function of the nervous system. In mouse, for example, functional expression of Stil is required for setting the midline and left-right polarity of the central nervous system $(\mathrm{CNS})^{15}$. In mutants which lack proper Stil expression and/or with defective structure of $S h h$ receptors, the embryos develop abnormally and die at age of E11. Using molecular genetic approaches, Izraeli et al. ${ }^{15}$ and Kasai et al. ${ }^{16}$ demonstrated that Stil functions in the Sonic hedgehog (Shh) pathway. Specifically, Stil regulates the transcription of Shh-target gene Gli1. Normally, GLI1 forms heterodimers after binding to cytoplasmic protein SUFU. The heterodimers cannot be translocated to the nucleus thereby the transcription of Gli1 gene is inhibited. When STIL is expressed, STIL binds SUFU, frees GLI1 from SUFU repression. Then, GLI1 can enter the nucleus, and gene transcription can start. If Stil is mutated, the transcription of Gli1 cannot be initiated (Fig. 1).

Recent studies have shown that Stil plays important role for proliferation, survival, regeneration, and possibly functions in dopaminergic (DA) neurons ${ }^{17,18}$. In humans, DA-mediated neural transduction is required for integration of sensory-motor signals and the control

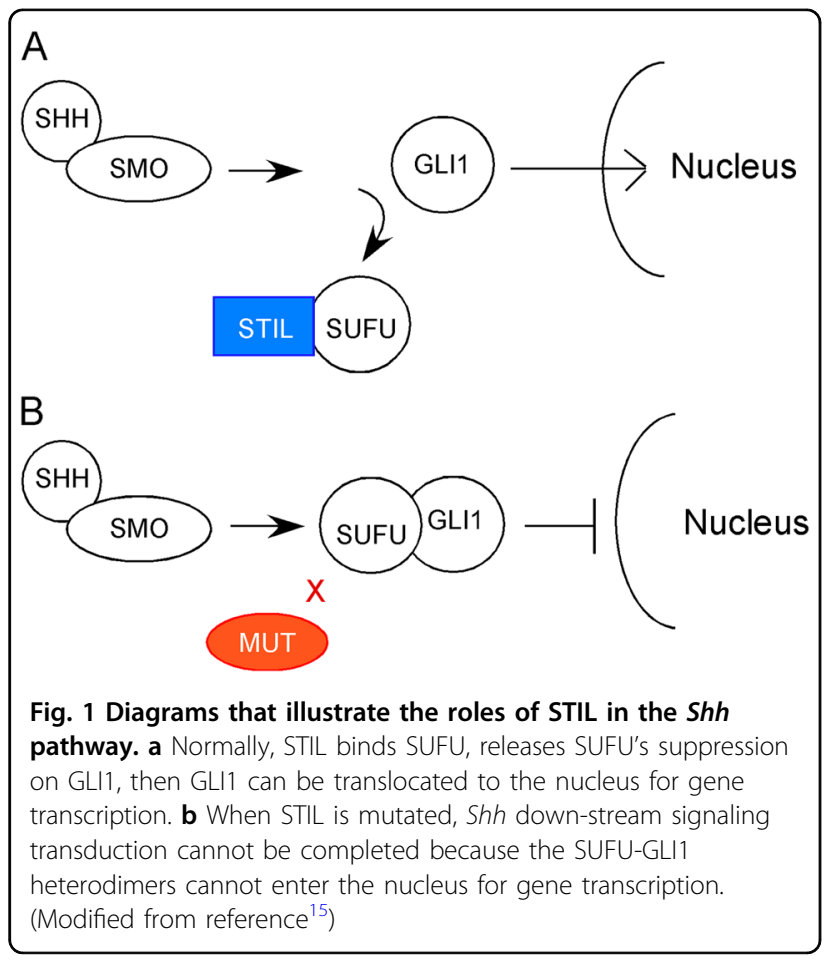

of movement ${ }^{19-22}$. The lack of DA cells and/or malfunction of DA neural networks may lead to a variety of neurological diseases, such as $\mathrm{PD}^{23-26}$. In this article, we will review some of the findings relate to the roles of Stil in DA cells. The data presented in this article are obtained from studies conducted in zebrafish models and cultured mammalian DA cell lines. The results may provide insight for future development of Stil as a potential bio-marker for better understanding the molecular and cellular mechanisms underlying human neurological disorders.

\section{Multi-function of STIL in DA cells \\ STIL protects DA cells}

While most the studies related to Stil were carried out using cultured cancer cell lines and mouse models $^{9,11,15,16,27-29}$, research that characterize Stil mutations that cause dramatic phenotypes (e.g., embryonic lethality, nervous system malfunction) can also be found by using other species, such as zebrafish. Pfaff et al. ${ }^{30}$ reported that in zebrafish, STIL is highly expressed in proliferating cells. During metaphase, STIL is concentrated in areas near the poles of the mitotic spindles. In cassiopeia (designated csp, which is a homolog of human Stil) mutants, the Stil gene is mutated, and the organization of spindle pole in proliferating cells is completely disrupted. Often, the mutants lack one or both centrosomes. As a consequence, the embryos develop abnormally and die between 7 and 10 days post-fertilization. In another study, $\mathrm{Li}$ and Dowling ${ }^{31}$ demonstrated that in zebrafish night blindness $b$ (designated $n b b$; also a homolog of human Stil) mutants, not only the development but also the function of the $\mathrm{CNS}$ are interrupted. In homozygous $n b b$ mutants, massive cell degeneration occurs in the developing brain, and the embryos die before 7 days post-fertilization. In heterozygous $n b b$ mutants, animals are viable but after 9 months of age, abnormalities are found in the CNS, e.g., the number of retinal DA cells is decreases, and the animals become night blind particularly after prolonged dark adaptation. In addition, in $n b b$ mutants the susceptibility of DA cells to neurotoxin is increased ${ }^{17}$, and the rate of DA cell regeneration is decreased ${ }^{32}$. Molecular cloning and sequence analysis of the $n b b$ locus revealed a nucleotide substitution at a donor-splicing site in the Stil transcript, which results in a predicted truncated peptide with 301 amino acids ${ }^{17}$.

To investigate if the expression of Stil is required for maintaining the DA cells (e.g., whether or not the decrease of DA cells in $n b b$ mutants is due to age-related neural degeneration), Li et al. ${ }^{17}$ examined the survival of DA neurons in zebrafish retinas in response to treatment with DA-specific neurotoxins, such as 6hydroxydopamine (6-OHDA) $)^{33,34}$. They found that deficiency in Stil expression led to increases in toxic 
susceptibility in DA neurons. In wild-type zebrafish, for example, treatment with sub-toxic levels of 6-OHDA $(0.33 \mu \mathrm{g} / \mu \mathrm{l})$ produced no obvious effects on the survival of DA cells (Fig. 2a, b). In $n b b$ mutants, however, the same treatment led to significant DA degeneration (Fig. 2c, d). The susceptibility of DA cells to neural toxins can be reversed by manipulating the levels of Shh signaling transduction, e.g., by inhibition of STIL expression using Stil-specific morpholinos (MOs), or increase of Shh signaling transduction by knocking-down SUFU expression with Sufu-specific MOs. In zebrafish, treatment with gene-specific MOs can efficiently knock-down their protein expressions ${ }^{35,36}$. In wild-type zebrafish, decreases in STIL expression (by Stil-MO) led to increases of the susceptibility of DA cell to sub-toxic levels of 6-OHDA. As a consequence, DA cell degeneration was observed when treated with sub-toxic levels of 6-OHDA (Fig. 2e). In contrast, in $n b b$ mutants, inhibition of SUFU expression (by Sufu-MO, which then lifted its suppression to GLI1, and increased Gl1 transcription) lowered the
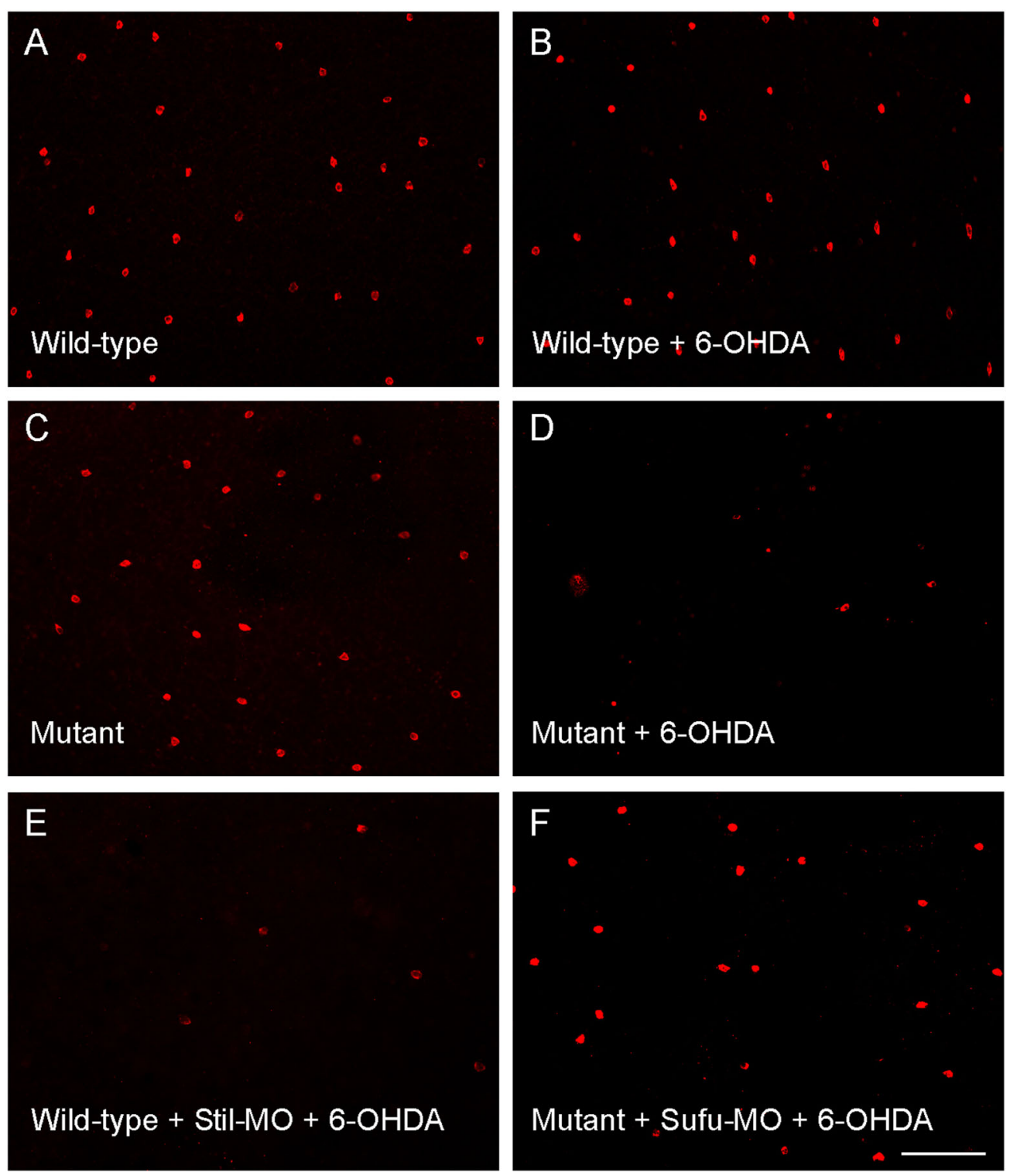

Fig. 2 Fluorescent images of flat-mounted zebrafish retinas that show the DA cells (labeled with antibodies against tyrosine hydroxylase) after treatment with sub-toxic 6-OHDA. $\mathbf{a}$, b Wild-type retinas that received sham or sub-toxic 6-OHDA treatment. No differences in the number of DA cells were observed. $\mathbf{c}$, $\mathbf{d}$ Mutant retinas that received sham or sub-toxic 6-OHDA treatment. The number of DA cells was decreased after drug treatment. e, f Wild-type and mutant retinas that received sub-toxic 6-OHDA injections, but previously treated with Stil- and Sufu-specific MOs, respectively. Note the increase of drug susceptibility (decreases in cell survival) in wild-type fish and the decrease of drug susceptibility (increases in cell survival) in mutant fish. Scale bar, $100 \mu \mathrm{m}$. (Modified from reference ${ }^{17}$ ) 
susceptibility of DA cells to neural toxins and protected the DA cells after drug treatment (Fig. 2f).

\section{STIL promotes DA regeneration}

Zebrafish are capable to replace lost neurons by reactivation of stem cell-like glia cells ${ }^{37-42}$. In a research aimed to distinguish whether or not the decrease of DA neurons in $n b b$ mutants is related to DA neural regeneration, Sun et al. (2014a) examined the proliferation of DA cells in adult zebrafish retinas after 6-OHDA induced neural degeneration. In this study, toxic levels of 6-OHDA $(5.0 \mu \mathrm{g} / \mu \mathrm{l})$ was applied in order to effectively induce the degeneration of DA cells. After a few days of 6-OHDA treatment, the samples were collected and labeled with antibodies against proliferating cell nuclear antigen (PCNA), a marker protein that only expresses in proliferating cells $^{38,43}$. In wild-type animals, after druginduced cell death of DA cells, robust regeneration of DA cells occurred, e.g., PCNA-positive cells could be readily identified (Fig. 3a). In $n b b$ retinas, however, in response to the same treatment, the rate of DA cell regeneration was decreased, e.g., the number of PCNApositive cells was only $30-40 \%$ of the number of PCNApositive cells counted in control retinas (Fig. 3b).

Because Stil functions in the Shh pathway, Sun et al. ${ }^{32}$ tested if the survival of DA cells is affected by Shh signaling transduction. The experiments were performed in wild-type animals in which the transduction of Shh signaling was blocked by steroidal alkaloid cyclopamine, and in $n b b$ mutants in which the transduction of Shh signaling was enhanced by knock-down of SUFU expression with Sufu-MO. Previous studies have demonstrated that cyclopamine, an antagonist of SMO receptor in the Shh pathway, can efficiently block the Shh signaling transduction and lower the transcription of Gli1 ${ }^{44,45}$. In wild-type fish, treatment with cyclopamine decreased the rate of DA cell regeneration after 6-OHDA induced degeneration (Fig. 3c). In Sufu-MO treated $n b b$ fish, after 6-OHDA treatment the number of cells that express PCNA-positive cells was increased to levels similar as seen in wild-type animals. Together, the data suggest that increases in Shh signaling transduction may rescue the defects in DA cell regeneration due to the expression of $n b b$ mutation (Fig. 3d).

\section{STIL induces mammalian DA cell proliferation}

The roles of STIL expression in mammalian DA cell proliferation were evaluated using cultured PC12 cells ${ }^{18}$. The PC12 cells were originally derived from rat adrenal pheochromocytoma cells ${ }^{46-48}$. Under normal culture conditions and with inductions by nerve growth factor (NGF), PC12 precursor cells can be proliferated and
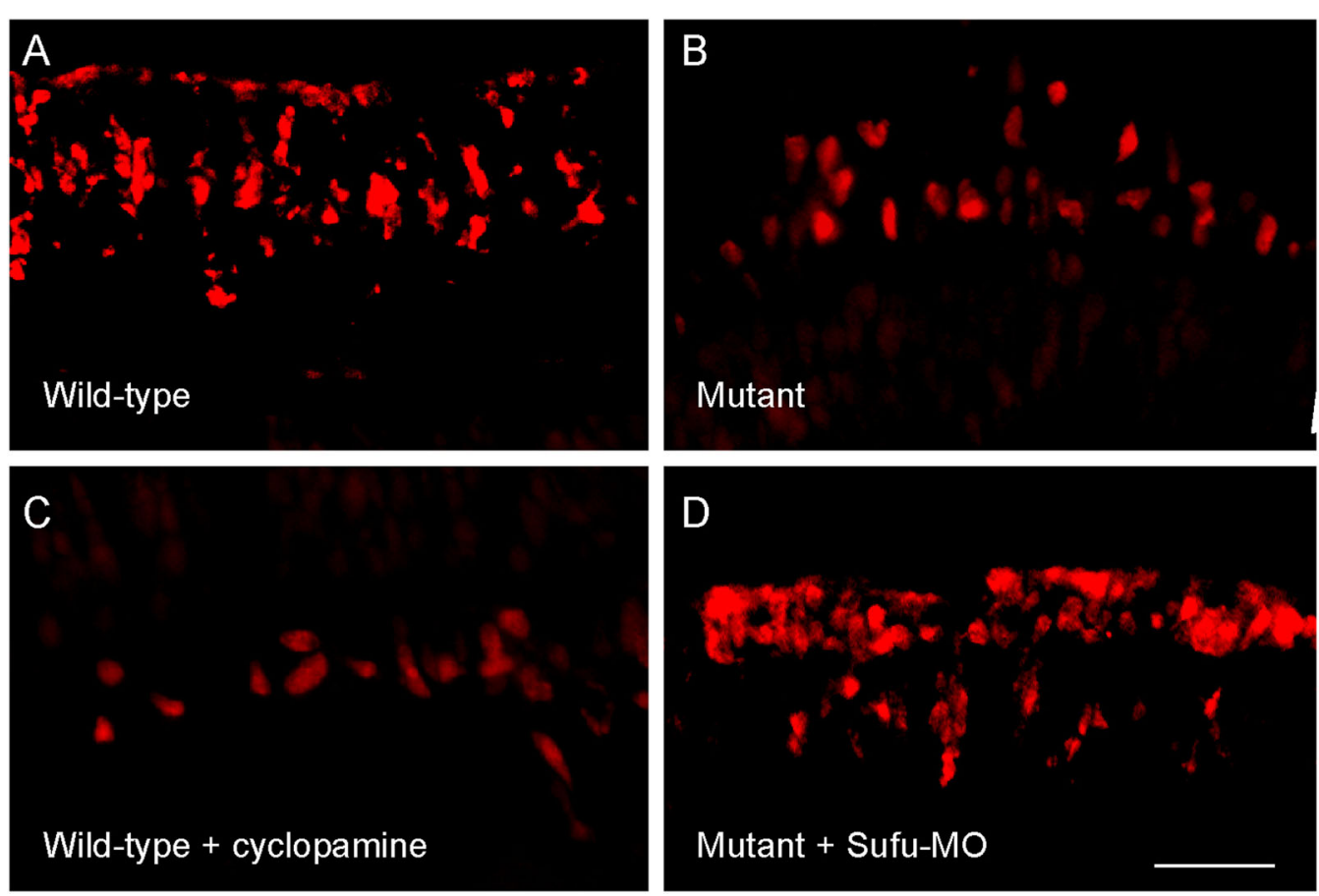

Fig. 3 Fluorescent images of retinal sections that show DA cell regeneration. $\mathbf{a}, \mathbf{b}$ Sections from wild-type and $n b b$ retinas. Note the decrease of PCNA labeling in the mutant. c A section from cyclopamine-treated wild-type fish. Note the decrease in the number of PCNA-positive cells. $\mathbf{d}$ A section from $n b b$ fish that received Sufu-MO injection. Note the increase in the number of PCNA-positive cells. Scale bar, $100 \mu \mathrm{m}$. (Modified from reference ${ }^{32}$ ) 
differentiated into DA cells ${ }^{49,50}$. The overexpression of Stil (by transfection with full-length human Stil sequence) increased the rate of cell proliferation of PC12 cells, by approximately $30 \%$ compared to control cells. In contrast, knock-down of Stil function (by Stil-specific shRNA) reduced the rate of $\mathrm{PC} 12$ cell proliferation, to a level approximately $45 \%$ of controls ${ }^{18}$.

To investigate whether or not STIL also functions in the Shh pathway as a de-repressor for proper signaling transduction in PC12 cells, Sun et al. ${ }^{51}$ examined the coexpression of STIL and Shh down-stream proteins, such as SUFU and GLI1, in PC12 cells. They found that both SUFU and GLI1 could be detected by anti-STIL antibody pull-down experiments. STIL and SUFU could also be detected by using anti-GLI1 antibody pull-down assays. These results provide evidence for the conserved roles of STIL in the Shh pathway in mammalian DA cells. The roles of Stil in Shh pathway in PC12 cells can also be demonstrated using pharmacological approaches. Carr et al. ${ }^{18}$ proposed that down-regulation of Shh signaling transduction by cyclopamine would mimic the proliferation defects caused by Stil-shRNA knock-down. They found that treatment with cyclopamine indeed reduced the proliferation rate of $\mathrm{PC} 12$ cells. Interestingly, overexpression of Stil was able to maintain a significant higher growth rate in the presence of cyclopamine. This suggests that when the initial Shh signaling is blocked, some of the down-stream signaling transduction could be continued by exogenous expression of STIL. That is, STIL binds SUFU and releases GLI1, then GLI1 enters the nucleus and starts gene transcription.

By the same token, whether or not the up-regulation of Shh signaling has a role on $\mathrm{PC} 12$ cell proliferation depends on the availability of STIL. This can be tested using pharmacologic approaches with purmorphamine while the expression of Stil is down- or up-regulated. It is known that purmorphamine functions as a SMO receptor agonist in the Shh pathway ${ }^{52-54}$. The application of purmorphamine increased the rate of PC12 cell proliferation $^{18}$. However, when the expression of Stil mRNA was blocked by Stil-shRNA, purmorphamine could not fully rescue the defects in PC12 cell proliferation ${ }^{18}$. That suggests that activation the up-stream Shh signaling transduction is not able to bring the proliferation rate to control levels if the expression of down-stream genes (e.g., $S t i l)$ is inhibited. Based on these observations, Carr et al. ${ }^{18}$ concluded that STIL may induce cell proliferation by propagating the intracellular Shh signal for subsequent transcription of Shh-target genes, which are cell cycle specific.

\section{STIL facilitates mammalian DA neural growth}

Carr et al. ${ }^{18}$ examined the distribution of STIL in PC12 cells. They found that STIL was expressed in both developing and NGF-induced mature cells. In differentiated mature cells, STIL was ubiquitous expressed in the cytoplasm, nucleus, as well as in the neurites. The result is not unexpected, considering the primary roles of Stil in mitotic entry, spindle pole organization, centriole formation and duplication. The expression of STIL in mature cells indicates novel non-proliferative functions of STIL in post-mitotic and differentiate cells, such as neural survival, neurite extension, synthesis or release of neurotransmitters. This supports the notion that Stil is involved in the regulation of microtubule elongation ${ }^{55}$.

While STIL is required for DA cell prolifaration and growth, the expression of Stil is not involved in PC12 cell neural differentiation. After NGF induction, out-growth of neurites was observed in PC12 cells regardless the levels of Stil expression. Also, the rate neurite growth was indistinguishable among the cells in which the expression of Stil was knocked-down (by transfection with StilshRNA) or the expression of Stil mRNA was up-regulated (by transfection with plasmids that contained the fulllength of human Stil DNA) ${ }^{18}$.

\section{STIL in regulation of DA cell drug susceptibility}

The effects of Stil expression on neural protection have been evaluated in cultured PC12 cells ${ }^{56}$. It appears that, to some extent, STIL may function as a "two-edged blade" that involves in both neural proliferation and degeneration, depends on the status of the cell. In proliferating PC12 cells, knock-down of STIL expression (by shRNA) produced no obvious effect on cells' drug toxicity, e.g., cell death occured when treated with high doses of 6-OHDA, similar as observed in control cells (Fig. 4a). However, overexpression of Stil (by transfection of full-length human Stil DNA) increased the susceptibility of PC12 cells to neurotoxins, and when exposed to higher doses of 6-OHDA (e.g., $50 \mu \mathrm{M}, 75 \mu \mathrm{M}$ ), excessive cell death was observed (Fig. 4b). In differentiated PC12 cells, Stil functions in opposite ways. That is, overexpression of Stil produced no effect on cell's drug susceptibility as compared to control cells, but knock-down of Stil increased the drug susceptibility and when treated with higher doses of 6-OHDA, the rate of cell death was increased as compared to control cells (Fig. 4c, d).

\section{Conclusion}

In summary, this paper provides evidence that through the Shh signaling transduction pathway the human oncogene Stil plays novel roles in DA cells. The expression of Stil is required for neural proliferation, survival, and regeneration. Considering the conserved roles of Stil in cell proliferation, the involvement of Stil in the Shh pathway, and the effect of Shh signaling in drug resistance, it is conceivable that Stil may function as an ON-OFF switch that controls the outcome of Shh signaling 

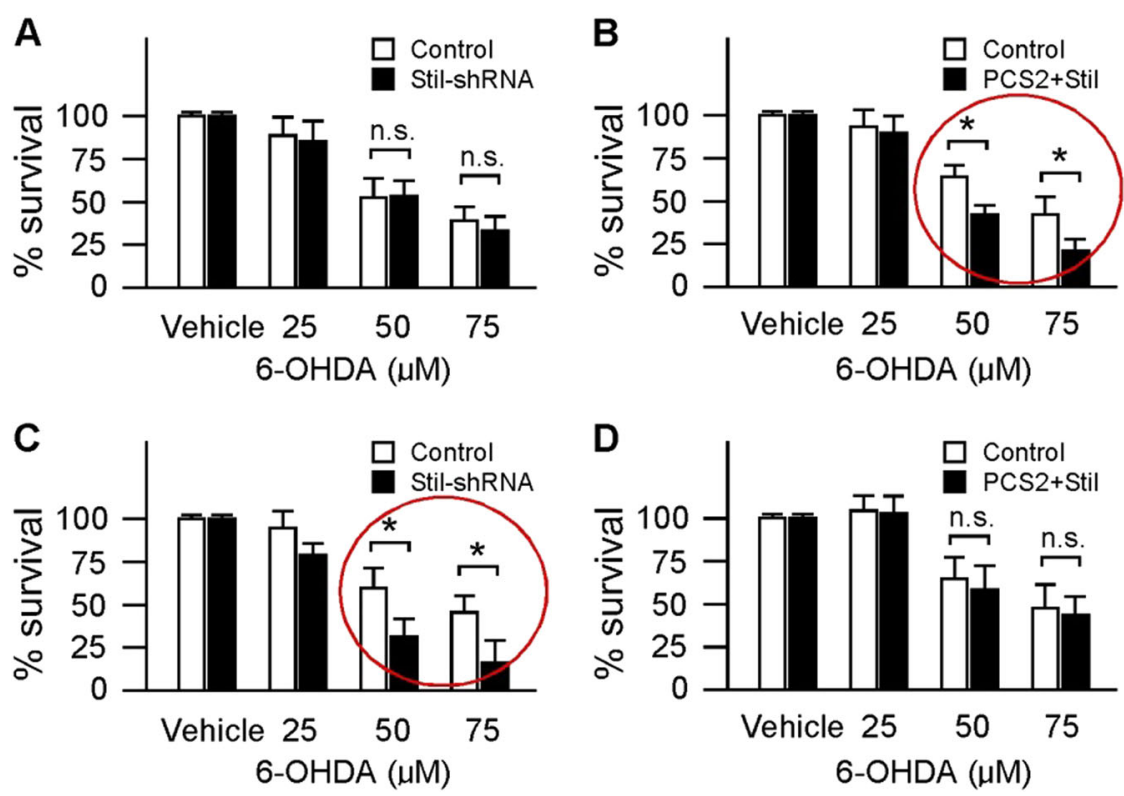

Fig. 4 Effects of Stil expression on PC12 cell drug susceptibility. The rate of survival under control conditions (treated with vehicle solution) is normalized to $100 \%$. a, b Percentage of cell survival after 6-OHDA treatment in proliferating cells. Note the increase of drug susceptibility after the overexpression of Stil (red circle). c, d Percentage of cell survival after 6-OHDA treatment in mature cells. Note the increase of drug susceptibility when the expression Stil is down regulated (red circle). Asterisks indicate statistical differences, $p<0.05$; ns, not significant (Modified from reference ${ }^{56}$ )

transduction, which in turns, regulates the fate of individual cell groups, for example, to be developed or undergo apoptosis. If this model proves true, then Stil may be considered as a bio-marker for basic and translational researches relate to human health, i.e., by analysis of the expression of Stil mRNA, the fate of certain cell types (e.g., cancer cells or neurons) can be predicted (e.g., proliferation, survival, or apoptosis). Future researches on Stil shall include but not limit to the characterization of the regulation of Stil expression in cancer cells and neurons, the interplay between Stil products and hormones that regulate cancer growth and neural degeneration, and the mechanisms of Stil-mediated Shh signaling transduction in drug resistance. At some point, one shall be able to control the molecular ON-OFF switch for Stil expression, and this will lead to either cell proliferation (e.g., neural regeneration) or apoptosis (e.g., cancer cells). It is expected that in the future Stil may be developed as a tool for diagnosis and/or treatment of human diseases.

\section{Acknowledgements}

Some of the works described in this review article were conducted in the author's labs and their researches were supported in part by grants from $\mathrm{NIH}$ (Grant\# R01EY013147) and NSFC (Grant\# 81671242).

\section{Author details}

'Department of Biological Sciences, University of Notre Dame, Notre Dame, IN 46556, USA. ${ }^{2}$ Center for Reproductive Medicine, Shandong University, Jinan 250001, China

\section{Conflict of interest}

The authors declare that they have no conflict of interest.

\section{Publisher's note}

Springer Nature remains neutral with regard to jurisdictional claims in published maps and institutional affiliations.

Received: 27 February 2019 Accepted: 18 March 2019

Published online: 25 April 2019

\section{References}

1. Aplan, P. et al. Disruption of the human scl locus by illegitimate V-(D)-J recombinase activity. Science 250, 1426-1429 (1990).

2. Aplan, P., Lombardi, D. \& Kirsch, I. Structural characterization of Sil, a gene frequently disrupted in T-cell acute lymphoblastic leukemia. Mol. Cell Biol. 11, 5462-5469 (1991).

3. Collazo-Garcia, N., Scherer, P. \& Aplan, P. Cloning and characterization of a murine SIL gene. Genomics 30, 506-513 (1995).

4. Karkera, J. et al. The genomic structure, chromosomal localization, and analysis of SIL as a candidate gene for holoprosencephaly. Cytogenet Genome Res $\mathbf{9 7}$, 62-67 (2002).

5. Stevens, N. R., Dobbelaere, J., Brunk, K., Franz, A. \& Raff, J. W. Drosophila Ana2 is a conserved centriole duplication factor. J. Cell Biol. 188, 313-323 (2010).

6. Andersen, J. S. et al. Proteomic characterization of the human centrosome by protein correlation profiling. Nature 426, 570-574 (2003).

7. Basto, R. et al. Flies without centrioles. Cell 125, 1375-1386 (2006)

8. Pelletier, L., OToole, E., Schwager, A., Hyman, A. A. \& Muller-Reichert, T. Centriole assembly in caenorhabditis elegans. Nature 444, 619-623 (2006).

9. David, A. et al. Lack of centrioles and primary cilia in STIL $(-/-)$ mouse embryos. Cell Cycle 13, 2859-2868 (2014).

10. Moyer, T., Clutario, K. M., Lambrus, B. G., Daggubati, V. \& Holland, A. J. Binding of STIL to Plk4 activates kinase activity to promote centriole assembly. J. Cell Biol. 22, 863-878 (2015).

11. Erez, A. et al. The SIL gene is essential for mitotic entry and survival of cancer cells. Cancer Res 67, 4022-4027 (2007). 
12. Kitagawa, D. et al. Spindle positioning in human cells relies on proper centriole formation and on the microcephaly proteins CPAP and STIL. J. Cell Sci. 124 3884-3893 (2011).

13. Arquint, C., Sonnen, K. F., Stierhof, Y. \& Nigg, E. A. Cell-cycle-regulated expression of STIL controls centriole number in human cells. J. Cell Sci. 125, 1342-1352 (2012).

14. Vulprecht, J. et al. STIL is required for centriole duplication in human cells. J. Cell Sci. 125, 1353-1362 (2012).

15. Izraeli, S. et al. Genetic evidence that Sil is required for the sonic hedgehog response pathway. Genesis 31, 72-77 (2001).

16. Kasai, K. et al. SCL/TAL1 interrupting locus derepresses GLI1 from the negative control of suppressor-of-fused in pancreatic cancer cell. Cancer Res $\mathbf{6 8}$, 7723-7729 (2008).

17. $L i$, J. et al. Functional expression of Stil protects retinal dopaminergic cells from neurotoxin-induced degeneration. J. Biol. Chem. 288, 886-893 (2013).

18. Carr, A. L. et al. The human oncogene SCL/TAL1 interrupting locus is required for mammalian dopaminergic cell proliferation through the sonic hedgehog pathway. Cell Signal. 26, 306-312 (2014)

19. Matsumoto, N., Hanakawa, T., Maki, S., Graybiel, A. M. \& Kimura, M. Role of corrected nigrostriatal dopamine system in learning to perform sequential motor tasks in a predictive manner. J. Neurophysiol. $\mathbf{8 2}$ 978-998 (1999).

20. Szczypka, M. S., Rainey, M. A. \& Palmiter, R. D. Dopamine is required for hyperphagia in Lep(ob/ob) mice. Nat. Genet 25, 102-104 (2000).

21. Djatchkova-Podkletnova, I. \& Alho, H. Alterations in the development of rat cerebellum and impaired behavior of juvenile rats after neonatal 6-OHDA treatment. Neurochem Res 30, 1599-1605 (2005).

22. Gengler, S., Mallot, H. A. \& Holscher, C. Inactivation of the rat dorsal striatum impairs performance in spatial tasks and alters hippocampal theta in the freely moving rat. Behav. Brain Res 164, 73-82 (2005).

23. Foltynie, T. \& Kahan, J. Parkinson's disease: An update on pathogenesis and treatment. J. Neurol. 260, 1433-1440 (2013).

24. Kalia, L. V. \& Lang, A. E. Parkinson's disease. Lancet 386, 896-912 (2015).

25. Wijemanne, S. \& Jankovic, J. Dopa-responsive dystonia--clinical and genetic heterogeneity. Nat. Rev. Neurol. 11, 414-424 (2015).

26. Tarakad, A. \& Jankovic, J. Diagnosis and management of Parkinson's disease. Semin Neurol. 37, 118-126 (2017).

27. Izraeli, S. et al. Expression of the SIL gene is correlated with growth induction and cellular proliferation. Cell Growth Differ. 8, 1171-1179 (1997).

28. Izraeli, S. et al. The SIL gene is required for mouse embryonic axial development and left-right specification. Nature 399, 691-694 (1999).

29. Castiel, A. et al. The stil protein regulates centrosome integrity and mitosis through suppression of chfr. J. Cell Sci. 124, 532-539 (2011).

30. Pfaff, K. L. et al. The zebra fish cassiopeia mutant reveals that SIL is required for mitotic spindle organization. Mol. Cell Biol. 27, 5887-5897 (2007).

31. Li, L. \& Dowling, J. E. Disruption of the olfactoretinal centrifugal pathway may relate to the visual system defect in night blindness b mutant zebrafish. J. Neurosci. 20, 1883-1892 (2000).

32. Sun, L. et al. The SCL/TAL1 interrupting locus (Stil) is required for cell proliferation in adult zebrafish retinas. J. Biol. Chem. 289, 6934-6940 (2014).

33. Li, L. \& Dowling, J. E. Effects of dopamine depletion on visual sensitivity of zebrafish. J. Neurosci. 20, 1893-1903 (2000).

34. Cronin, A. \& Grealy, M. Neuroprotective and Neuro-restorative Effects of minocycline and rasagiline in a zebrafish 6-Hydroxydopamine model of Parkinson's disease. Neurosci 367, 34-46 (2017).

35. Bill, B. R., Petzold, A. M., Clark, K. J., Schimmenti, L. A. \& Ekker, S. C. A primer for morpholino use in zebrafish. Zebrafish 6, 69-77 (2009).
36. Bedell, V. M., Westcot, S. E. \& Ekker, S. C. Lessons from morpholino-based screening in zebrafish. Brief. Funct. Genom. 10, 181-188 (2011).

37. Raya, A., Consiglio, A., Kawakami, Y., Rodriguez-Esteban, C. \& Izpisúa-Belmonte, J. C. The zebrafish as a model of heart regeneration. Cloning Stem Cells $\mathbf{6}$ 345-351 (2004).

38. Vihtelic, T. S., Soverly, J. E., Kassen, S. C. \& Hyde, D. R. Retinal regional differences in photoreceptor cell death and regeneration in light-lesioned albino zebrafish. Exp. Eye Res 82, 558-575 (2006).

39. Raymond, P. A., Barthel, L. K., Bernardos, R. L. \& Perkowski, J. J. Molecular characterization of retinal stem cells and their niches in adult zebrafish. BMC Dev. Biol. 6, 36 (2006).

40. O'Reilly-Pol, T. \& Johnson, S. L. Melanocyte regeneration reveals mechanisms of adult stem cell regulation. Semin Cell Dev. Biol. 20, 117-124 (2009).

41. Perlin, J. R., Robertson, A. L. \& Zon, L. I. Efforts to enhance blood stem cell engraftment: Recent insights from zebrafish hematopoiesis. J. Exp. Med 214, 2817-2827 (2017)

42. Lindsey, B. W. et al. The role of neuro-epithelial-like and radial-glial stem and progenitor cells in development, plasticity, and repair. Prog. Neurobiol. 170, 99-114 (2018).

43. Fimbel, S. M., Montgomery, J. E., Burket, C. T. \& Hyde, D. R. Regeneration of inner retinal neurons after intravitreal injection of ouabain in zebrafish. $J$. Neurosci. 27, 1712-1724 (2007).

44. Dunn, M., Mercola, M. \& Moore, D. Cyclopamine, a steroidal alkaloid, disrupts development of cranial neural crest cells in Xenopus. Dev. Dyn. 202, 255-270 (1995).

45. Taipale, J. et al. Effects of oncogenic mutations in Smoothened and Patched can be reversed by cyclopamine. Nature 406, 1005-1009 (2000).

46. Greene, L. A. \& Tischler, A. S. Establishment of a noradrenergic clonal line of rat adrenal pheochromocytoma cells which respond to nerve growth-factor. Proc. Natl. Acad. Sci. USA 73, 2424-2428 (1976).

47. Greene, L. A. \& Rein, G. Release of $[3 \mathrm{H}]$ norepinephrine from a clonal line of pheochromocytoma cells (PC12) by nicotinic cholinergic stimulation. Brain Res 138, 521-528 (1977).

48. Rebois, R., Reynolds, E., Toll, L. \& Howard, B. Storage of dopamine and acetylcholine in granules of Pc12, a clonal pheochromocytoma cell-line. Biochemistry 19, 1240-1248 (1980).

49. Park, Y. H., Kantor, L., Wang, K. K. \& Gnegy, M. E. Repeated, intermittent treatment with amphetamine induces neurite outgrowth in rat pheochromocytoma cells (PC12 cells). Brain Res 951, 43-52 (2002).

50. Yamboliev, I. A., Smyth, L. M., Durnin, L., Dai, Y. \& Mutafova-Yambolieva, V. N. Storage and secretion of beta-NAD, ATP and dopamine in NGF-differentiated rat pheochromocytoma PC12 cells. Eur. J. Neurosci. 30, 756-768 (2009).

51. Sun, L. et al. Characterization of SCL/TAL1 interrupting locus (Stil) mediated Sonic hedgehog (Shh) signaling transduction during mammalian dopaminergic cell proliferation. Biochem Biophy Res Comm. 449, 444-448 (2014).

52. Sinha, S. \& Chen, J. Purmorphamine activates the hedgehog pathway by targeting smoothened. Nat. Chem. Biol. 2, 29-30 (2006).

53. Lauth, M., Bergstrom, A., Shimokawa, T. \& Toftgard, R. Inhibition of GLImediated transcription and tumor cell growth by small-molecule antagonists. Proc. Natl. Acad. Sci. USA 104, 8455-8460 (2007).

54. El-Akabawy, G., Medina, L. M., Jeffries, A., Price, J. \& Modo, M. Purmorphamine increases DARPP-32 differentiation in human striatal neural stem cells through the hedgehog pathway. Stem Cells Dev. 20, 1873-1887 (2011).

55. Comartin, D. et al. CEP120 and SPICE1 cooperate with CPAP in centriole elongation. Curr. Biol. 23, 1360-1366 (2013).

56. Li, L. et al. A novel function of the human oncogene Stil: Regulation of PC12 cell toxic susceptibility through the Shh pathway. Sci. Rep. 5, 16513 (2015). 